\title{
Impacto en las redes sociales de las Grandes Empresas Españolas: Reputación Corporativa, Integridad y Comportamiento Ético
}

Impact on social networks Spanish Major Companies: Corporate Reputation, Integrity and Ethical Behaviour

Casado, A. M.

Dpto. de Comunicación

ESIC Business \& Marketing School ana.casado@esic.edu

Peláez, J. I.

Dpto. de Lenguajes y Ciencias de la Computación Universidad de Málaga jignacio@lcc.uma.es

\section{Callejón, A. M.}

Dpto. de Finanzas y Contabilidad

Universidad de Málaga

amcallejon@uma.es
Casado, A. M., Peláez, J. I. y Callejón, A. M. (2014). Impacto en las redes sociales de las Grandes Empresas Españolas: Reputación Corporativa, Integridad y Comportamiento Ético. Revista Internacional de Investigación en Comunicación aDResearch ESIC. № 9 Vol 9. Primer semestre, enero-junio 2014. Págs. 100 a 117 DOI: 10.7263/ADRESIC.009.006 


\section{RESUMEN}

\section{Clasificación JEL: \\ M31, M32, 033 \\ Palabras clave:}

Comportamiento Ético,

Reputación Corporativa,

Social Media,

Redes Sociales

La reputación corporativa, RC, es actualmente un intangible de gran valor para las empresas. Su identificación, medición y gestión efectiva resultan claves para la supervivencia empresarial y su sostenibilidad en el tiempo. Pero esta gestión no es sencilla, ya que la relación con los publicos esta cambiando debido a las nuevas tecnologías, y por los componentes que forman parte de la misma, la responsabilidad social corporativa, las relaciones laborales, cuestiones medioambientales, imagen y marca, y por supuesto la ética empresarial, ya que en un momento como el actual, dominado por la desconfianza, la integridad y el comportamiento ético de las compañias se consideran los pilares fundamentales para sustentar dicha RC. Es por ello, que es preciso analizar la relación existente entre la RC y sus diferentes componentes.

En este trabajo se realiza un estudio donde se analiza la relación existente entre la integridad y la RC percibida por los públicos en las redes sociales y en los medios on-line. Para ello se utiliza una muestra de empresas españolas seleccionadas atendiendo a su mayor capitalización. Los resultados de este estudio permiten conocer en qué grado la ética de las grandes compañías influye en su RC y si tal percepción recibida a través de las redes sociales penaliza o no el atractivo o admiración que sienten por las mismas en los social media. Esta asociación ofrecerá una nueva perspectiva de cómo la Integridad se desenvuelve en el escenario actual de relaciones entre la empresa y sus públicos a través del entorno on-line. En definitiva, esta investigación muestra si Ciudadano y Empresa, persona humana y persona jurídica, actúan y expresan su conducta en las redes sociales bajo una misma escala ética. Igualmente, ayuda a comprobar la predisposición que tienen —uno de los dos o los dos - a contribuir en la descontaminación y recuperación de la confianza perdida. Sólo así podremos mantener una RC sólida en un entorno global tan complejo y cambiante en el que el ser humano, en calidad de persona física o jurídica, es el protagonista.

ABSTRACT

\section{Jel Classification: M31, M32, 033 Key words:}

Ethical Behaviour, Corporate Reputation, Social Media, Social Networks
Corporate Reputation, CR, is currently a high intangible value for companies. Its recognition, measurement and effective management are the key factors to corporate survival and sustainability over time. But this effort is not simple, the relationship with their stakeholders is changing due to new technologies, labor relations, environmental issues, corporate image, branding and business ethics. Therefore, it is necessary to analyze the relationship between the $\mathrm{CR}$ and its various components.

This paper presents a study where the relationship between integrity and CR perceived by the public in social networks and media on-line is analyzed. For this purpose a sample of Spanish companies were selected according to their profit size. The results of this study allow us to understand into what extent the ethics of large companies manage to influence their perception and whether or not this CR received through social networks penalize their attractiveness or admiration in the social media. This partnership will provide a new perspective on how integrity performs in the current scenario between companies and their stakeholders over Internet. Ultimately, this research shows if citizens and companies, human person and legal person, act and express their behaviour in social networks under the same ethical level. It also helps to prove the tendency —one of the two or both — to contribute to the improvement and recovery of the lack of trust in the business market. Only then can we maintain a solid CR in a complex and changing global environment in which the human being, as a natural or legal person, is the protagonist. 


\section{Introducción}

Movidos en gran medida por la diversidad de costumbres que advertían en los distintos pueblos, los filósofos griegos analizaron con hondura qué tipos de comportamientos contribuían al desarrollo del ser humano y cuáles, por el contrario, impedían ese crecimiento o deterioraban a la persona. Surgieron así los primeros esbozos del saber ético, que en Sócrates, Platón y, sobre todo, Aristóteles, alcanzan un grado de madurez considerable. Según Aristóteles, la ética orienta al hombre y le ayuda a desarrollar con coherencia todas las potencialidades que hay en su interior (su dáimon), dando como resultado una "vida lograda" (eu-demonía).

Sin embargo, sólo a finales del siglo XVIII, con el inicio del capitalismo industrial, surgen los debates acerca de la responsabilidad social y la ética de las empresas (Cacioppe et al, 2008): la ética se incorpora entonces, de manera generalizada, al mundo económico, y se hace presente en todos sus estratos. La crisis financiera que surge en EEUU a mediados del año 2007, y que pronto se extiende por todo el mundo, hace que el interés por esta materia se acreciente, llegándose a cuestionar si esta crisis tiene su verdadero origen en razones puramente financieras o más bien éticas. La cuestión fue ya apuntada en sus orígenes (Colander, Goldberg, Haas, Juselius, Kirman, Lux y Sloth, 2009) y desarrollada más tarde con argumentos sólidos (Carroll y Buchholtz, 2011).

En este contexto, los gobiernos de las naciones aprueban normativas contra la corrupción y fomentan la responsabilidad social (RSC) en la gestión de las empresas, la transparencia de información y el uso responsable del poder, con objeto de establecer unos mínimos niveles de conducta ética entre los integrantes de la comunidad económica. El conjunto de normas resul- tantes, puede ser una medida buena e incluso necesaria, pero siempre resultará insuficiente. En palabras del profesor Argandoña (2011: 28) "La ética en una organización no consiste sólo o fundamentalmente en la aplicación de normas, códigos o principios establecidos desde fuera, ni en una valoración de los costes y beneficios de cada decisión. No responde sólo a una racionalidad económica, porque en la vida real, las decisiones van mucho más allá de la elección de medios para conseguir fines dados: los fines mismos son objeto de elección".

En este sentido, además de confundir los fines con los medios, se han tratado de justificar éstos con aquellos, instalándose en el quehacer económico, ciertos comportamientos, impuestos primero por la pura facticidad, legitimados por su uso común y casi universal. Según observaron algunos autores (Andreaus, Vaccaro y Aßländer, 2012; Morata, 2010) estas prácticas, carentes de principios éticos, nos han conducido a la situación actual. Algunas de estas prácticas reiteradas han sido: (1) la irresponsabilidad de la comercialización de productos financieros, que han facilitado y potenciado un consumo desmesurado, que a su vez ha generado una cascada de insolvencias, con graves y dolorosas consecuencias para los consumidores; (2) la especulación en los mercados financieros, para maximizar los lucros propios; (3) la codicia de los altos directivos financieros, que utilizan una falsa ética, conocida de ordinario como ética de la intención (soy bueno porque proyecto buenas intenciones); (4) la irresponsabilidad de las empresas, que han focalizado su atención en la inmediatez de un beneficio máximo a toda costa; (5) la irresponsabilidad de los gobiernos, que ceden su gestión a las empresas y se doblegan a los intereses particulares de estas, en detrimento del bien 
común; (6) un consumo no responsable por parte del ciudadano/consumidor; (7) la irresponsabilidad de los medios de comunicación, movidos por los intereses puramente económicos de su propia editorial; y (8) la inmediatez de las redes sociales, masivamente utilizadas como referencia principal ante cualquier decisión relativa a la empresa (compra, consumo e inversión).

Ciertamente, esto ha ocurrido siempre, y pese a la puesta en marcha de numerosos mecanismos y la predisposición de entidades públicas y privadas hacia un comportamiento más ético, seguimos muy cerca del punto de partida. ¿Por qué? La respuesta está en la propia persona: en lo que es, en lo que dice y en lo que hace. En su Integridad. No olvidemos que una empresa debe ser entendida como un conjunto de personas que trabajan ordenadamente para conseguir unos mismos objetivos finales. En este sentido el profesor Melendo (1990) afirma que en el inicio y en el término de toda actuación empresarial se encuentran personas humanas; las empresas son organizaciones de personas, entre personas y para personas. Y son precisamente esas personas las que construyen a través de sus valores personales, la cultura empresarial, con un mayor o menor compromiso con la ética. Las personas que tienen unos principios morales universales están más dispuestas a dar importancia a la ética en la empresa como garantía de éxito (Vitell y Ramos, 2006).

Como consecuencia, podemos establecer una cierta correlación entre los valores personales de los presidentes que han liderado una compañía y la RC que se ha generado con cada uno de ellos. Un ejemplo claro es lo que publica el Ranking de RC MERCO (2012): "ocho de las 10 empresas con mejor RC tienen a sus líderes entre los 10 más reputados". En la medida en que es- tos valores personales se incorporan como principios de actuación en el desempeño de la actividad profesional, no por imposición sino por convicción (Joyner y Payne, 2002), las empresas construirán, sobre unos cimientos sólidos, una verdadera RC sostenida en el tiempo, basada en la confianza, el respeto y por tanto en su integridad. Sus actitudes, creencias, comportamientos conformarán la cultura empresarial que irá impactando y sucediéndose en las siguientes generaciones. En este mismo sentido en las pequeñas compañías, la cultura empresarial, los valores y por tanto la ética de la organización quedan firmemente establecidos por sus fundadores (Joyner y Hofer, 1992).

Los diferentes stakeholders, y en general la opinión pública, podrán o no percibir este comportamiento ético. Puede ocurrir, que la imagen que la empresa desea proyectar en sus comunicaciones y acciones no se corresponda con la percepción que de ella se tiene. Estas diferencias pueden incluso percibirse como incoherencias, faltas de integridad, desestabilizando así las relaciones de confianza empresa-públicos, generando un gap ético de difícil tratamiento que pueda derivar hacia una grave pérdida de RC.

A partir de este análisis situacional, se establece la siguiente hipótesis para este trabajo:

Hipótesis: La integridad de las empresas favorece la RC percibida por los stakeholders en las redes sociales y en medios on-line.

La presente investigación, por tanto, pretende avanzar en este campo de investigación, con las siguientes aportaciones: (1) Dar a conocer al tejido empresarial del mercado español cuáles son los atributos de integridad que más impactan en la mejora de su RC; (2) Analizar con antelación los conflictos éticos para poder diagnosticar a tiempo real los posibles riesgos de actuación que 
pueden impactar en la RC. El aporte específico pasa por aprovechar la instantaneidad que las redes sociales ofrecen para extraer esas opiniones de los stakeholders y afrontar las consecuencias exponenciales que opiniones dispersas (geográfica y moralmente) puedan provocar. (3) Un soporte filosófico donde no se considera la ética desde la perspectiva conceptual, sino desde la acción.

Este trabajo se estructura de la siguiente forma. En primer lugar se profundiza en el marco teórico donde se abordan los principales conceptos en los que se centra esta investigación, así como los estudios empíricos más relevantes sobre Ética y RC. Posteriormente se presenta la metodología empleada y se analizan los resultados obtenidos. Por último, se sintetizan las principales conclusiones.

\section{Revisión de la literatura}

Algunos estudios han puesto de relieve que la globalización de los mercados y la irrupción de las TIC están obligando a las empresas a buscar un nuevo camino para garantizar su sostenibilidad en el tiempo y su diferenciación en los mercados (Aras y Crowther, 2010; Millar, Hind y Maga, 2012; Miron, Petcu y Sobolewschi, 2011).

Estamos en la era del conocimiento, donde las redes sociales han cambiado las relaciones entre las organizaciones y sus públicos. Hoy son los públicos quienes expresan y validan el comportamiento de una entidad y, por lo tanto, garantizan su continuidad (Celaya, 2008; Ritter, 2009; Schau y Gilly, 2003). Una percepción negativa sostenida en el tiempo puede incluso desplazarla del mercado donde opera.

Por otro lado los productos o servicios que se ofertan son prácticamente similares a los de la competencia. El escenario ha cambiado. En la década de los 80, el 65\% del valor de una compañía dependía de sus activos tangibles y el 35\% de sus intangibles (Kendrick 1994). Actualmente, intangibles como la RC, la RSC o el comportamiento ético suponen el 70\% del valor de una empresa, y el 30\% lo constituyen los tangibles (Daum 2003).

En este marco, diversos autores muestran que los activos intangibles son los principales recursos de diferenciación que deben ser orientados hacia la gestión del conocimiento y del capital intelectual: Nonaka (1991, 1994); Nonaka y Takeuchi (1995); Brooking (1996); Edvinsson \& Malone (1997); Roos et al. (1997); Sveiby (1997); Teece (2000) y Martín-Torres (2006). Diversos trabajos evidencian que estos activos tienen una importancia creciente para las empresas, como sustento de sus ventajas competitivas, ya que sus características específicas les hacen tener un fuerte potencial diferenciador respecto de los competidores (Zabala et al. 2005; Cravens, Goad Oliver y Ramamoorti, 2003; Roberts y Dowling, 2002; Mehralian et al., (2013). Waslh y Wiedmann (2004) y Kunle y Ajao (2013). Todos ellos consideran que la buena o mala gestión de la RC es un determinante del éxito o fracaso corporativo, por lo que debería considerarse como un activo de mercado.

Además, actualmente existe una pérdida de confianza por parte de los consumidores, empleados, comunidad en general hacia las empresas e instituciones (Colander et al., 2009). Esta pérdida de credibilidad viene provocada por las malas prácticas, los comportamientos no éticos y la falta de integridad entre lo que se hace y lo que se dice. De ahí que dos autores hayan establecido una clara relación entre comportamiento ético y sostenibilidad empresarial (Carrol y Buchholtz, 2011). 
La construcción de relaciones sostenibles sólo será posible si los públicos tienen una percepción favorable del comportamiento de las compañías en el tiempo; es decir, siempre que estas tengan una buena RC. La buena o mala RC, como intangible, puede sumar o restar al valor de esa empresa y puede mejorar o reducir su confianza (Riel, 2012).

\subsection{Integridad, Ética y RC}

En la literatura existente, el concepto de integridad se relaciona con la unidad interna que se establece entre el modo de ser, el modo de pensar y el modo de actuar; por el contrario, la falta de integridad denota una ruptura interna dentro de la empresa. No existe unidad entre lo que la empresa es y lo que proyecta. A veces se mira más hacia afuera que hacia adentro, y como decían los clásicos, para ver bien, muchas veces hay que cerrar los ojos.

En este sentido, la identidad cede paso al reconocimiento, y se intenta por todos los medios proyectar lo que se cree que al cliente le gustaría que la empresa fuera, en detrimento de la propia realidad de la empresa.

La integridad de la empresa ha de ir precedida por la integridad de las personas que la componen y ser una consecuencia de ella. La superioridad en el ser, propia de la persona humana, hace que los diversos elementos que la constituyen formen una unidad; pero, además - y esto nos interesa particularmente - también lleva consigo una exigencia, un deber: libremente, cada persona debe instaurar una unidad en todo su obrar, debe obrar con coherencia, evitando las contradicciones entre su modo de ser y su manera de obrar y entre unos comportamientos y otros.

Al contrario, cuando las personas adolezcan de falta de unidad o de coherencia, la empresa verá también mermada su integridad.
Por su parte, el concepto de ética puede entenderse como la ciencia que se ocupa de descubrir las reglas que permiten al hombre pasar de ser como es a ser como debería ser según su verdadero fin, descubierto por la inteligencia (Aristóteles). Esta definición nos obliga a establecer con rigor lo que es la empresa, como sujeta a ética, y lo que es la persona, como esencia de la empresa.

Así pues, hablar de ética es hablar de ética de la persona humana. No cabría una ética empresarial al margen de la ética de las personas, las cuales conforman el principal activo de la empresa. “... el perfeccionamiento máximo de los miembros que la componen [...] constituye un imperativo ético que se impone a la empresa por su misma naturaleza intrínseca de organización humana; y no [...] una especie de sobreañadido caritativo, con el que los empresarios podrían 'poner la guinda' a una actividad económica más o menos recta" (Melendo, 1990: 37). Todo individuo que forma parte de una empresa, antes de ser trabajador, es persona. Tiene la empresa una prioritaria dimensión humana, que impide concebirla exclusivamente como un simple generador de renta. Sin embargo, no debe entender el lector que estas dos dimensiones se excluyen entre sí, sino todo lo contrario: se complementan y enriquecen, a veces, cediendo paso la una a la otra. Podemos decir que la ética personal precede a la ética profesional/empresarial.

Avanzar en este sentido requiere de una conceptualización de lo que entendemos por persona y por empresa. Como sabemos, el término "persona" empezó a utilizarse a comienzos de nuestra era para indicar la grandeza que corresponde a todo ser humano, con independencia absoluta de cualquier otra circunstancia que el hecho - no "simple", sino "sublime" - de tra- 
tarse de un ser humano. Por eso, también desde hace siglos, el término "persona" tiene dos connotaciones fundamentales: la "dignidad" y la "singularidad" o "unicidad" (cada persona es única e irrepetible). Al denominarlo "persona", se indica que todo ser humano es siempre un fin $y$, como consecuencia, que nunca debe tratarse como un mero medio o instrumento para conseguir otros objetivos que su propio desarrollo o perfeccionamiento.

Desde tal punto de vista, "el fin de la empresa consiste en promover la mejora humana de cuantos con ella se relacionan y de la sociedad en su conjunto, mediante la gestión económica de los bienes y servicios que genera y distribuye, y de los que naturalmente se siguen unos beneficios con los que logra también subsistir ly crecer] como empresa" (Melendo 1990: 27).

En la literatura que rodea al Business Ethics, hay un gran debate abierto en la definición de "los valores de una empresa", sin que tampoco haya consenso a la hora de determinar las dimensiones o componentes que definen la ética en los negocios (corporativa). Por ejemplo, Víctor y Cullen (1998) entienden las siguientes dimensiones de la ética empresarial: atenciones, leyes y códigos, reglas, instrumentación e independencia; por su parte, Treviño et al. (1998) presentan 10 dimensiones, que son: el entorno ético, el enfoque en los trabajadores, la atención a la ciudadanía, la obediencia a la autoridad, la implementación de códigos, (Chun et al. 2013).

No coincidimos con algunos autores (Kaptein y Van Dalen, 2000) que pretenden agrupar los dominios de las practicas éticas en tres bloques: (1) las relaciones de la empresa con los stakeholders externos,(2) la ética del funcionamiento externo y (3) la ética en el comportamiento de los empleados. Desde nuestro punto de vista, la éti- ca corporativa será la consecuencia de la implantación de los valores éticos de las personas que conforman la empresa, aplicados éstos al desempeño (acciones y comunicaciones) de la organización con sus diferentes stakeholders y con la ciudadanía en general.

Como afirma Joyner y Payne (2002), la realidad es que la empresa no puede subsistir sin la sociedad, por lo cual aquella debe ser más sensible a las demandas éticas de los ciudadanos y a las expectativas de comportamiento honesto que estos esperan de ella.

A su vez, la empresa necesita la colaboración de la sociedad. La exigencia de una ética bien vivida no es sólo responsabilidad de la empresa como única unidad económica, pues el sistema económico en su conjunto también está formado por las unidades de consumo — las familias - y las administraciones.

Por otro lado, la exigencia de un comportamiento ético puede tener su origen en dos ámbitos bien distintos. Uno es el de la ética de la intención, que surge con el deseo de convencer a los stakeholders de que se están haciendo las cosas bien, por imposición o por falta de integridad. "La ética no se identifica solo con las acciones que miran a los efectos de las acciones en los demás: decir la verdad puede ser un acto ético, pero también puede no serlo, si se hace por cálculo, estrategia, temor o para engañar. No se trata de conseguir un fin externo, sino que es la condición para perseguir otros fines" (Argandoña, 2011: 26).

Pero también puede tener su origen en la ética de la acción, que surge por el deseo de hacer las cosas bien por convicción, una ética gestionada correctamente desde dentro de la empresa. Ésta sería, desde nuestra opinión, la ética bien entendida; y en consecuencia, bien vivida. La 
ética empieza y termina en la persona, y así se recoge en los issues de confianza más relevantes.

Hay siempre una relación contractual entre la empresa y el resto de sus stakeholders. Por tanto, se debe medir la ética en ambas partes: si no se tiene la misma escala ética, —es decir, si ambas éticas no están debidamente alineadas- es muy difícil que pueda surgir un entendimiento ético y que la actuación de aquella sea siempre percibida como "éticamente correcta". La importancia que la ética tiene en una compañía ha sido discutida desde muy diferentes puntos de vista (McMurrian y Matulich, 2006). Sin embargo, el interés que despierta el estudio de la ética en la empresa, casi desde sus comienzos, —años 50 del pasado siglo — ha venido marcada por el interés en conocer la relación entre la ética y el beneficio. Esa relación es, para la mayoría de los autores, positiva en el ámbito empresarial (Orlitzky, Schmidt y Rynes, 2003; Waddock y Graves, 1997; Chun, Shin, Choi y Kim, 2013), mientras que para otros resulta negativa (Bowie, 1998). Estos últimos afirman que la ética y el beneficio están inversamente relacionadas. Así, McMurrian y Matulich (2006) afirma en su estudio empírico, que hay todavía muchas empresas que aún que prácticas empresariales no éticas no serán descubiertas; y, por tanto, no tendrán consecuencias negativas en la compañía.

Finalmente, y en referencia a la RC, son muchas las definiciones existentes en la literatura. Para Riel y Fombrun (2007: 43-44) la RC es "la evaluación global de la organización realizada por sus stakeholders. Esta evaluación se basa en las percepciones de los stakeholders relativas a la capacidad de la organización para lograr sus expectativas". Para Roberts y Dowling (2002: 1077-1093), la RC es "la percepción de las acciones pasadas y las perspectivas futuras de una compañía la cual describe el atractivo global para todos los participantes cuando se compara con otras compañías". Schultz et al. (2001: 24-41) consideran que es "el juicio de una empresa realizado por un conjunto de audiencias sobre la base de las percepciones y valoraciones". Para Waddok (2000: 323-345), la RC es "la capacidad percibida de la organización para satisfacer las expectativas de los stakeholders". Por su parte, Wartick (2002: 371-392) afirma que "la RC es la agregación de las percepciones de cada uno de los participantes sobre cómo la organización responde y satisface las demandas y expectativas de los stakeholders de la organización".

En el ámbito académico son muchas las investigaciones y autores que afirman que la RC es un intangible que aporta una ventaja competitiva y provee de múltiples beneficios: reduce costes (Roberts y Dowlings, 2002), crea una barrera a la competencia (Dolphin, 2004; Davies et al, 2003), multiplica el valor de las marcas (Black et al, 2000; Fan, 2005), fortalece los productos y servicios y constituye un escudo frente a la crisis (Villafañe, 2004; Dowling, 1986, 1994), transmite compromiso de calidad y confianza a los consumidores (Fombrun y Shanley, 1990; Bauer et al, 2003; Berens et al, 2005; Casado et al., 2013), facilita el acceso a nuevos mercados (Fombrun y Riel, 2004), proporciona éxito financiero (Fombrun, 2001; De Quevedo et al, 2005), disminuye el impacto frente a una crisis y promueve la recuperación (Eccles et al, 2007).

Para una gestión adecuada de la RC, se deben definir: (1) Cómo se construye la RC; y (2) Qué variables son las causas que la originan.

La RC se construye a partir de la coherencia en la percepción que los diferentes stakeholders tienen entre lo que lo que la empresa es -misión, visión y valores (Identidad)—, lo que dice- 
comunicación- y lo que hace-desempeño- (Riel y Fombrun, 2007; Riel, 2012). Y se expresa a través de cuatro atributos emocionales: Estima, Respeto, Admiración y Confianza.

Las dimensiones que se trabajan en el modelo más estandarizado de gestión de la RC, Reptrak, utilizado por las empresas más reputadas en el mercado español son: (1) Oferta Producto y Servicio; (2) Innovación; (3) Trabajo; (4) Integridad; (5) Ciudadanía (RSC); (6) Liderazgo; y (7) Finanzas.

Dentro del concepto Integridad, propuesto en Reptrak, se distinguen las siguientes variables: Comportamiento Ético, Transparencia Informativa y Uso Responsable del Poder (Riel y Fombrun, 2007). En este modelo la RSC se entiende desde la perspectiva del apoyo a la ciudadanía en causas sociales, contribución a la sociedad (mecenazgos, etc.) y protección al medioambiente, si bien, desde nuestro punto de vista, la RSC no se puede acotar a estas tres variables. Además entendemos que se puede dar una cierta ambigüedad al interpretar los datos de medición de variables tan poco mensurables como es la RSC; así se puede caer en la simplificación de medir el grado de compromiso de una empresa por el número de acuerdos suscritos con proyectos humanitarios, por el importe en euros que suponga una ayuda social. Es decir, se corre el riesgo de reducir la RSC al mero cumplimiento de unos mínimos recogidos en los informes GRI.

Podemos encontrar empresas que realizan grandes inversiones en RSC con el único fin de dar un valor añadido a su marca y no como parte de su gestión basada en un desempeño ético e integro con todos sus stakeholders.

Sin embargo la Integridad, tal y como se define en el modelo, se construye a partir del pensar y el actuar de la persona en un mismo sentido: comportamiento ético, transparencia informativa y uso responsable del poder.

Desde esta perspectiva, tanto la Integridad como el Comportamiento Ético y la RC se construyen en base a un mismo principio: la coherencia entre lo que se dice y se hace.

\subsection{Evidencia empírica de la relación entre la Ética y la RC}

Son pocos los estudios empíricos existentes que relacionan integridad, ética y RC. Y no se ha encontrado ninguno que relacionen estas tres variables en el contexto de las redes sociales y medios on-line. Algunos autores, con sus trabajos empíricos, relacionan la práctica de la ética con la percepción que los stakeholders tienen de ella. En este sentido, encontramos estudios empíricos en los que se han puesto en relación los valores éticos de una empresa (su desempeño) y la percepción que los públicos tienen de ellos. Vitell y Ramos (2006) demuestran que la implantación de los valores éticos dentro de la cultura empresarial y el grado de compromiso no sólo de sus empleados sino también de sus directivos, influyen positivamente en el éxito de la empresa. Además, los factores personales y organizacionales tienen un impacto en las percepciones que los públicos tienen sobre el comportamiento ético de éstas.

En otros estudios, la relación se establece entre los códigos éticos implementados en una compañía y la percepción que los públicos tienen de ella. Singh (2011) revela que son cinco las variables que más impactan en una percepción favorable de la implantación de dichos códigos en las empresas. Éstas son: La intención de establecer un código; implementación del código; aplicación de los procesos de comunicación para el seguimiento y control del código interno; 
plan de comunicación externa para la difusión del código ético; la utilidad de dicho código ético.

Profundizando en la relación entre ética y RC, Bendixen y Abratt (2007) focalizan el estudio en las relaciones entre los proveedores y el departamento de compras de la empresa: la percepción de los primeros respecto al desempeño ético de las actuaciones de los segundos. Las conclusiones muestran que: (1) los principios éticos de actuación de los departamentos de compras son las dimensiones subyacentes de las percepciones éticas que tienen los proveedores, (2) que dichas percepciones éticas impactan en la RC que el proveedor tiene sobre el comprador y (3) Se presenta un modelo de RC basado en la gestión y relaciones éticas entre comprador y proveedor.

Finalmente, Alonso (2009) relaciona la RC o confianza de los stakeholders, a través de la transparencia que las empresas tienen en internet, confirmando que las variables que más contribuyen a proporcionar esa transparencia corresponden a las empresas con mayor capitalización, que están presentes en más de un mercado y ponen a disposición de los accionistas medios electrónicos para su participación. Además, el sector que más transparencia proporciona es el sector de petróleo y energía y los que influyen de manera contraria son el de servicios financieros e inmobiliarios.

\section{Metodología}

\subsection{Muestra}

El presente trabajo de investigación utiliza una muestra de 42 empresas seleccionadas aleatoriamente entre la población de grandes corporaciones españolas, activas a 30 de abril de 2013 y con un total activo superior a 400 millones de euros. Las características de dicha muestra y su distribución sectorial aparecen en la tabla 1. La obtención de información se refiere al período comprendido entre el 8 de abril y el 8 de mayo de 2013, lo que ha supuesto disponer finalmente de un total de 1302 observaciones para cada una de las variables del estudio (Tabla 1).

\subsection{Variables}

Para la presente investigación se han construido dos índices, uno de RC y otro de Integridad, a partir de diferentes indicadores obtenidos de los análisis previos (Riel y Fombrun, 2007). Los índices seleccionados y los atributos que los definen, aparecen en la tabla 2. Los valores de dichas variables se han formado con las percepciones online extraídas diariamente de la red social Twitter, de las noticias de los principales diarios

\section{Tabla 1 - Distribución de la muestra por sectores}

\begin{tabular}{|l|c|c|c|c|}
\hline Sector & $\begin{array}{c}\text { Número de } \\
\text { Empresas }\end{array}$ & SIC & $\begin{array}{c}\text { \% de Empresas } \\
\text { Públicas }\end{array}$ & $\begin{array}{c}\text { Presencia } \\
\text { en Bolsa }\end{array}$ \\
\hline Petróleo y Energía & 7 & 13 & 14.28 & 85.72 \\
\hline Industria & 9 & $16,20-39$ & 0.00 & 100.00 \\
\hline Comercio, Transpy Serv. & 14 & $40-49,50-51$ & 21.42 & 42.86 \\
\hline Comunicaciones & 4 & 48 & 0.00 & 75.00 \\
\hline Financiero & 8 & $60-67$ & 0.00 & 100.00 \\
\hline Total & 42 & & & \\
\hline
\end{tabular}


digitales españoles y de foros empresariales en internet, entre el 8 de abril y el 8 de mayo de 2013.

\section{Tabla 2 • Índices de RC e Integridad}

\begin{tabular}{|l|l|}
\hline Índice de RC & Índice de Integridad \\
\hline Estima & Transparencia \\
\hline Respeto & Uso responsable del poder \\
\hline Admiración & Comportamiento ético \\
\hline Confianza & \\
\hline
\end{tabular}

Los procesos de captura de información y análisis que se han llevado a cabo en las diferentes fuentes de información, han sido los siguientes: En el caso de Twitter, se realiza un filtrado previo a través de su interfaz de búsqueda utilizando como palabra clave las marcas corporativas de cada empresa, como también los posibles nombres utilizados por los usuarios de esta red para referirse a cada una de ellas (sinónimos). Después de este filtrado se han capturado para este estudio un total de 83.871 tweets. Estos tweets se asocian directamente con las empresas sobre las que se opina y se insertan en la base de datos para ser analizados.

Respecto a los medios de comunicación de masas, se accede diariamente a los principales portales web nacionales con mayor notoriedad y relevancia en la red. Para este estudio se han capturado un total de 348 noticias referidas a la muestra de empresas. A partir de los enlaces RSS de cada uno de ellos, se accede a un listado con la agenda setting de noticias diarias, se realiza una lectura automatizada del titular de cada noticia y si dicho titular contiene la marca o sinónimos de la empresa se accede al texto de la noticia a través de su URL y se inserta la información en la base de datos para su posterior análisis.
Por último, en el caso de los foros, se han analizado un total de 12 foros especializados en diferentes temáticas (economía, empresas, bancos y cajas, seguros y consumo) y dirigidos a diferentes públicos. Para este estudio se han capturado un total de 5.951 posts referidos a la muestra de empresas. En cada uno de los foros se publican diariamente una serie de temas de opinión, los usuarios generan un post cada vez que intervienen en un tema. A través de nuestra propia herramienta de captura se extrae la información de dichos posts y se filtra por marca o sinónimo de empresa. Una vez seleccionados, se insertan en la base datos para ponerlos a disposición del motor de análisis.

El análisis semántico de la información almacenada de los tweets, noticias online y posts, está basado en una metodología que utiliza diccionarios de datos y patrones de búsqueda. Inicialmente se han utilizado más de 350 expresiones etiquetadas que generan expresiones regulares. Estas expresiones regulares son los patrones de búsqueda para clasificar la información de cada una de las empresas en los respectivos atributos. En nuestro caso de estudio, las expresiones se clasifican según se refieran a Integridad (Transparencia, Comportamiento Ético y Uso responsable del poder) y a RC (Estima, Respeto, Admiración y Confianza). Una vez realizada dicha clasificación, se establece el tono (positivo, negativo o neutro) de dichas expresiones asociados a los atributos por cada empresa. En tercer lugar, se genera un registro diario por empresa donde se contabiliza el total de issues positivos, negativos o neutros por cada atributo.

Para el cálculo del índice cuantitativo de las percepciones online extraídas diariamente a través de estas tres fuentes - Twitter, noticias online y foros- se ha construido un algoritmo que 
considera un amplio número de criterios, entre ellos se contemplan: (1) El rango varia de 0 (información completamente negativa) a 10 (información completamente positiva); (2) La ventana de datos para incorporar el histórico en el índice diario es de 15 días; (3) El peso de las informaciones de cada uno de los días decrece de forma lineal en función de la antigüedad; y (4) La distribución del número de informaciones pondera en el cálculo del índice diario.

De otra parte, y con objeto de evaluar la capacidad predictiva de la integridad de las empresas, incluimos un número de variables de control en nuestro análisis (tabla 3). Primero, el tamaño de la empresa, que ha sido utilizado como variable para controlar la tendencia de empresas más grandes para conseguir una mayor RC. Las empresas más grandes tienen un mayor número de accionistas, inversores y terceros pendientes de su evolución, por eso necesitan ofrecer información que contribuya a la comprensión de las empresas a los agentes interesados (Marston y Polei, 2004). Además, las grandes compañías son más visibles y en consecuencia, tienen más costes políticos (Watts y Zimmermann, 1978). El tamaño de la empresa se ha computado por el logaritmo natural del total activo del balance, porque el efecto del tamaño en la RC podría ser no lineal y una misma cantidad de incremento de activo podría tener una influencia más grande en la RC cuándo las empresas son pequeñas.

También, y para controlar los efectos de las estructuras económico-financieras de las empresas en los diferentes sectores de la economía, se ha creado una variable dummy que clasifica la industria en 5 sectores Verrecchia (1983), puso de manifiesto que los costes políticos pueden variar de un sector a otro. No obstante la evidencia empírica, aunque sugiere una relación, no parece confirmar con rotundidad la relación entre el sector y la disposición de información y mecanismos de participación a través de Internet (Bonsón y Escobar, 2002).

Finalmente, se ha tenido en cuenta que la presencia de una empresa en mercados ajenos al nacional de referencia incentiva la disposición de información de la empresa para reducir las asimetrías informativas que puede provocar un accionariado y terceros dispersos entre distintos países (Portes y Rey, 2000). Internet puede proporcionar de forma instantánea información a distintos mercados y reducir los costes de participación social (Sengupta, 1998). Para controlar los efectos de esta presencia en mercado internacionales se ha incluido una variable dummy que toma el valor 1 si la compañía tiene presencia en mercados internacionales y que toma el valor 0 en caso contrario.

\section{Tabla 3 - Definición de las variables de control}

\begin{tabular}{l|l}
\hline Variable & Definición \\
\hline Total Activos & Logaritmo natural del total activo al cierre del ejercicio $2012^{*}$ \\
\hline Sector (1 al 5) & Variable dummy que toma el valor 1 si pertenece al sector y 0 en caso contrario \\
\hline Internacionalidad & $\begin{array}{l}\text { Variable dummy que toma el valor } 1 \text { la empresa tiene presencia en mercados internacionales } \\
\text { y } 0 \text { en caso contrario }\end{array}$ \\
\hline *2011 para empresas no cotizadas & \\
\hline
\end{tabular}




\section{Resultados}

La tabla 4 muestra una descripción estadística de las variables a través de la media, la desviación estándar, el valor máximo y el valor mínimo. Los resultados obtenidos muestran una baja dispersión respecto a los valores medios. Además, la variable con más valor asignado por los stakeholders es la transparencia (7,37 de media). Por su parte, la variable que hace referencia al tamaño de las empresas, medido por el total activo, confirma que la muestra seleccionada corresponde a compañías de gran dimensión (más de 15.000 millones de euros de activos medios). es una variable significativa y que presenta relación directa con la RC (coeficiente $=0.191$ y significativa al nivel del 0.05). Entonces, se verifica la hipótesis de que a mayor nivel de integridad percibida on-line, mayor es también la percepción on-line de RC. Además, dos variables de control han resultado incluidas en el modelo, Internacionalización y Sector, ambas con coeficientes negativos. Esto último permite confirmar que las empresas que centran su actividad sólo en territorio nacional, y en sectores más próximos a las actividades primarias y secundarias, tienen un mayor valor de RC. Por su parte, el

\section{Tabla 4. Variables estadísticas descriptivas}

\begin{tabular}{|l|c|c|c|c|}
\hline Variable & \multicolumn{2}{|c|}{ Significado } & Desv. Estándar & Max. \\
\hline RC & 6.17 & 1.01 & 7.86 & 4.55 \\
\hline Integridad & 5.93 & 2.16 & 9,97 \\
\hline Transparencia & 7.37 & 3.69 & 10.00 & 0.59 \\
\hline Uso responsable del poder & 5.98 & 2.17 & 1.03 \\
\hline Comportamiento ético & 5.87 & 2.18 & 9,53 \\
\hline Total Activos & 15433325,83 & 23375549,48 & 9.77 \\
\hline Total Activo en $€ .103 ;$ Indice $[0,10]$ & & 93118712,00 & 604063,00 \\
\hline
\end{tabular}

Con el objetivo de determinar el grado de significatividad de la dimensión integridad en la variabilidad del índice de $\mathrm{RC}$, se ha construido un modelo de regresión múltiple, aplicando para ello el procedimiento stepwise regression ${ }^{1}$. De esta forma se evita la colinealidad y se seleccionan las variables independientes que más influyen en el índice de RC (tabla 5). Se observa que la integridad percibida por los stakeholders

1 Es un método de regresión por etapas "hacia delante", en el que la entrada y salida de las variables en la ecuación de regresión se realiza a partir de los valores del estadístico t-Student (Stevens, 1996). coeficiente de determinación del modelo es de 0,224 , lo que significa que estas variables seleccionadas, en conjunto, explican un $22.4 \%$ de la varianza del índice de RC.

Además, se ha estudiado cuáles son las variables que más influyen en la construcción de cada una de las dimensiones de la integridad percibida (Tabla 6). Los resultados obtenidos señalan que todos los atributos estudiados en relación con la integridad son variables significativas para explicar el nivel de RC percibida, y que, a excepción del atributo transparencia, las empresas de 


\section{Tabla 5 • Análisis de Regresión. Variable Dependiente: Índice de RC}

\begin{tabular}{|c|c|c|c|c|}
\hline Variables & Coeficientes & Error Estándar & t-stat & $\operatorname{Pr}>|t|$ \\
\hline Constante & 4.331 & 0.676 & 5.645 & $0.000^{* * * *}$ \\
\hline Integridad & 0.191 & 0.082 & 2.314 & $0.026^{* *}$ \\
\hline Internacionalización & -0.801 & 0.386 & -0.076 & $0.045^{* *}$ \\
\hline Sector & -0.252 & 0.144 & -1.742 & $0.090^{*}$ \\
\hline $\begin{array}{l}\mathrm{R} 2=0.224 \quad \mathrm{R} 2 \mathrm{Aj} \cdot=0.162 \\
* * * \text { significativo al } 0.01\end{array}$ & $\begin{array}{r}\text { timación }=1.133 \\
\quad * \text { significativo }\end{array}$ & $\mathrm{FPr}=0.021$ & Durbin Watson $=1.506$ & \\
\hline
\end{tabular}

\section{Tabla 6 • Análisis de Regresión por los atributos de Ética. Variable dependiente: Índice de RC}

\begin{tabular}{|c|c|c|c|c|}
\hline Variables & Coeficientes & Std. Error & t-stat & $\operatorname{Pr}>|t|$ \\
\hline Constante & 5.222 & 0.457 & 11.427 & $0.000^{* * *}$ \\
\hline Transparencia & 0.135 & 0.056 & 0.354 & $0.021^{* *}$ \\
\hline
\end{tabular}

\section{Atributo :Transparencia}

\begin{tabular}{|l|c|c|c|c|}
\hline Variables & Coeficientes & Std. Error & t-stat & Pr>|t| \\
\hline Constante & 4.343 & 0.770 & 5.640 & $0.000^{* * *}$ \\
\hline Uso responsable del poder & 0.188 & 0.082 & 2.276 & $0.029^{* *}$ \\
\hline Internacionalización & -0.806 & 0.387 & -2.082 & $0.044^{* *}$ \\
\hline Sector & -0.251 & 0.145 & -1.732 & $0.091^{*}$ \\
\hline
\end{tabular}

$R 2=0.221 \quad R 2 \quad A j .=0.159$ error est. de estimación $=1.136 \quad F=3.584 \quad F P r .=0.022 \quad$ Durbin Watson $=1.502$ **** significativo al $0.01{ }^{* *}$ significativo al $0.05{ }^{*}$ significativo al 0.10

Atributo: Uso responsable del poder

\begin{tabular}{|c|c|c|c|c|}
\hline Variables & Coeficientes & Error Estándar & t-stat & $\operatorname{Pr}>|t|$ \\
\hline Constante & 4.329 & 0.763 & 5.671 & $0.000^{* * *}$ \\
\hline Comportamiento ético & 0.192 & 0.082 & 2.340 & $0.025^{* *}$ \\
\hline Internacionalización & -0.796 & 0.385 & -2.066 & $0.046^{* *}$ \\
\hline Sector & -0.253 & 0.144 & -1.755 & $0.087^{*}$ \\
\hline $\begin{array}{l}R 2=0.226 \quad \mathrm{R} 2 \quad \mathrm{Aj}=0.165 \\
* * * \text { significativo al } 0.01\end{array}$ & $\begin{array}{l}\text { stimación }=1.132 \\
0.05 \quad * \text { significativ }\end{array}$ & $\mathrm{FPr}=0.020$ & rbin Watson= 1.507 & \\
\hline
\end{tabular}

\section{Atributo: Comportamiento Ético}


ámbito local y en sectores más básicos de la actividad económica son las que mayor respeto, admiración, confianza o estima proporcionan.

El análisis realizado ha estudiado la asociación entre la integridad percibida on-line por los stakeholders y los niveles de RC que asignan a las grandes empresas españolas. Además, el análisis se ha extendido a los atributos que determinan dicha integridad. Los modelos de regresión muestran que dichos atributos, individualmente considerados, también resultan significativos para explicar la variabilidad de la RC. Estos resultados son similares a los obtenidos al medir la RC y la integridad con otras técnicas de medición como las encuestas (Riel y Fombrun, 2007).

Finalmente, y en relación a la variable tamaño de las empresas, en ninguno de los modelos ha resultado significativa, contrariamente a lo que obtenido en otras investigaciones, en las que las empresas más grandes y que operan en mercados no nacionales, mostraban niveles más altos de RC (Ettredge et al., 2002; Xiao et al., 2004).

\section{Conclusiones}

Una vez finalizado el estudio empírico así como el marco teórico en el cual se centra, las conclusiones a las que llegamos con este trabajo son que se puede dar por aceptada la hipótesis de que la integridad de las empresas favorece la RC percibida por los stakeholders y captada a través de las fuentes on-line. Además, y de forma particular, dos variables de control, internacionalización y sector, han permitido confirmar que las empresas que centran su actividad sólo en territorio nacional y en sectores más próximos a las actividades primarias y secundarias tienen un mayor valor de RC.

Por último, el análisis se ha extendido a los atributos que determinan la integridad. Los modelos de regresión muestran que dichos atributos, individualmente considerados, también resultan significativos para explicar la variabilidad de la RC.

\section{Agradecimientos}

Este trabajo se enmarca dentro del proyecto de investigación TIN2011-26046 del programa de ciencia y tecnología del Ministerio de Ciencia e Innovación. 


\section{Bibliografía}

Aristóteles. Ética a Nicómaco, Lib. I.

Argandoña, A. (2011). "La ética y la toma de decisiones en la empresa", Universia Business Review, 2, 22-31.

Aras G. y Crowther, D. (2010). "Sustaining business excellence", Total Quality Management \& Business Excellence, $21(5), 565-576$

Alonso, M.M. (2009). "Transparencia de las empresas para la confianza de los stakeholders a través de internet: Un análisis empírico", Hitos de Ciencias Económico Administrativas, $43,105-114$.

Andreaus, Vaccaro y Aßländer (2012). "Which values and which organizations. Introduction to the special isse of the EBEN AC 2010 Conference", Journal of Business Ethics, 106, 1-3. DOI:10.10007/s10551-011-113-5.

Bendixen, M. y Abratt, R. (2007). "Corporate identity, ethics and reputation in supplier-buyer relationships", Journal of Business Ethics, 76, 69-82. DOI: 10.1007/s10551-0069273-4.

Black, E.L., Carnes, T.A. and Richardson, V.H. (2000). "The marketing valuation of corporate reputation", Corporate Reputation Review, 31(1), 31-41.

Bauer, H., M. Hammerschmidt, M. and Braehler, M. (2003). "The Customer lifetime value concept and its contribution to corporate valuation", Yearbook of Marketing and Consumer Research, 1 .

Berens, G., Riel, van C.B.M. and Bruggen, G.H. (2005). "Corporate associations and consumer product responses: the moderating role of corporate brand dominance", Journal of Marketing, 69(July), 35-48.

Bonsón, E. y Escobar, T. (2006). "Digital reporting in eastem Europe: An empirical studies", International Journal of accounting information systems, 7, 299-318.

Brooking, A. (1996). Intelectual Capital. Core Asset for the Third Millenium Enterprise. London, International Thomson Business Press.

Cacioppe, R.; Forster, N. y Fox, M. (2008). "A survey of Manager's Perceptions of Corporate Ethics and Social Responsibility and Actions that may Affect Companies 'Success", Journal of Business Ethics, 82, 681-700. DOI: 10.1007/ s10551-007-9586-y.
Carroll, A.B. y Buchholtz, A.K. (2011). Business E society: Ethics, sustainability, and stakeholder management. Mason (Ohaio, USA), South-Western Cengage Learning.

Casado, A.M., Méndiz, A. and Peláez, J.I. (2013). "The evolution of Dircom: from communication manager to reputation strategist", Communication E Society, 26(1), 47-66.

Celaya, J. (2008). La empresa en la Web 2.0. Madrid, Gestión 2000 .

Chun, J., Shin, Y., Choi, J. y Kim, M. (2013). "How does corporate ethics contribute to firm financial performance? The mediating role of collective organizational commitment and organizational citizenship behavior?", Journal of Management,39(4),853-877. DOI: 10.1177/0149206311419662.

Colander, A., Goldberg, M., Haas, A., Juselius, K; Kirman, A., Lux, T. y Sloth, B. (2009). "The financial crisis and the systemic failure of the economics pression", A Journal of politics and society, 21(2-3), 249-267.

Cravens, K., GoadOlver, E. y Ramamoorti, S. (2003). "The Reputation Index: Measuring and Managing Corporate Reputation", European Management Journal, 21(2), 201-212.

Davis, G., Chun, R., Silva da R.V. y Roper, S. (2003). Corporate Reputation and Competitiveness. London, Routledge.

Daum, J. H. (2002). Intangible Assets and Value Creation. Chichester, Wiley.

De Quevedo, E.; De la Fuente, J. M. y Delgado, J. B. (2005). "Reputación Corporativa y Creación de Valor. Marco Teórico de Una Relación Circular", Investigaciones Europeas de Dirección y Economía de la Empresa, 11(2), 81-97.

Dolphin, R. (2004). "Corporate Reputation - a Value Creating Strategy", Corporate Governance, 4, 77-92.

Dowling, G.R. (1986). "Managing your corporate images", Industrial Marketing Management, 15, 109-115.

Dowling, G.R. (1994). Corporate Reputations: Strategies for Developing the Corporate Brand. London: Kogan Page.

Eccles, R., Newquist, S. y Schartz, R. (2007). "Reputation and its Risks", Harvard Business Review, 85(2), 104-114.

Edvinsson, L. y Malone, M. (1997). Intellectual Capital: Realizing your company's true value by finding its hidden brainpower. Nueva York, Happer Collins. 
Ettredge, M., Richardson, V.J., y Scholz, S. (2002). "Dissemination of information for investors al corporateWeb sites", Journal of accounting \& public policy, 21, 357-69.

Fan, Y. (2005). "Ethical branding and corporate reputation", Corporate Communications: An International Journal, 10(4), 341-350.

Fombrun, C.J. (2001). "Corporate Reputation as economic assets", en R.E. Freeman Mitt y J.S. Harrison (eds) The Blackwell Handbook of Strategic Management, Blackwell, USA, 293.

Fombrun, C. J. y Shanley, M. (1990). "What's in a Name?: Reputation Building and Corporate Strategy", Academy of Management Journal, 33(2), 233-258.

Fombrun, C.J. and Riel, van C.B.M. (2004). Fame \& Fortune. How successful companies build winning reputations. New York, Pearson Education.

Joyner, B. y Hofer, C. (1992). "The day tasks of successful venture creation and development" en Douglas Naffziger and Jeffrey Hornsby (eds.), Emerging Entrepreneurial Strategies in the 1990's, Conference Proceedings of the Seventh Annual National USASBE Conference, Chicago. IL, Ball State University, 239-253.

Joyner, B. y Payne, D. (2002). "Evolution and implementation: a Study of Values, Business Ethics and Corporate Social Responsibility", Journal of Business Ethics, 41, 297-311.

Kaptein, M. y Van Dalen, J. (2000). "The empirical assessment of corporate ethics: A case study", Journal of Business Ethics, 24, 95-114.

Kendrick, J.W. (1994). "Total capital and economic growth". Atlantic Economic Journal, 22(1), 1-8.

Kunle, L.P. y Ajao, R. (2013). "Corporate Reputation as a strategic asset", International Journal of Business and Social Science, 4(2), 220-225.

Marston, C.L. y Polei, A. (2004). "Corporate reporting on the Internet by German companies", International Journal of accounting information system, 5, 285-311.

Martín-Torres, M.R. (2006). "A procedure to design a structural and measurement model of Intellectual Capital: an exploratory study". Information \& Management, 43(5), 617-626.

McMurrian, R. C. y Matulich, E. (2006). "Building customer value and profitability with business ethics", Journal of Business \& Economics Research (JBER), 4(11), 11-17.

Melendo, T. (1990). Las claves de la eficacia empresarial. Madrid, Ed. Rialp.
Mehralian, G., Rasekh, H.R., Akhawan, P. et al. (2013)." Prioritization of intellectual capital indicators in knowledge-based industries: Evidence from pharmaceutical industry", International Journal of Information Management, 33(1), 209-216.

Miron D., Petcu, M and Sobolevschi, I.M. (2011). "Corporate Social Responsibility and the sustainable competitive advantage", Amfiteatru Economic, 12(29), 162-179.

Millar, C., Hind, P. and Maga, S. (2012), " "Sustainability and the need for change: organizational change and transformational visión", Journal of Organizational Change Management, 25(4), 489-500.

Morata, F. (2010). "Responsabilidad e irresponsabilidad social en tiempos de crisis", en Francesc Morata, Blanca Villà y Carmeb Suárez (eds.): La Responsabilidad de la Empresa a debate: lecciones de la crisis, Barcelona, Institut Universitari d'Etudis Europeus, 11-19.

Nonaka, I. (1991). "The Knowledge Creating Company", Harvard Business Review, 69(6), 96-104.

Nonaka, I. (1994). "A Dynamic Theory of Organizational Knowledge Creation", Organization Science, 5(5), 14-37.

Nonaka, I. y Takeouchi. H. (1995). The Knowledge-Creating Company: How Japanese Companies Create the Dynamics for Innovation. Nueva York, Oxford University Press.

Orlitzky, M.; Schmidt, F y Rynes, S. (2003): "Corporate Social Financial Performance: A Meta-Analysis", Organization Studies,24(3),403-441.DOI:10.1177/0170840603024003910.

Portes, R. y Rey, H. (2000). "The determinants of crossborder equity flows”. NBER. Papeles de trabajo No. W7336. Oficina Nacional de Investigación Económica. Cambridge, Massachussets.

Riel, C.B.M. van \& Fombrun, C.J. (2007). Essentials of Corporate Communication. Implementing practices for effective reputation management. New York: Routledge.

Riel van C.B.M (2012). Alinear para ganar. Madrid, Lid Editorial Empresarial.

Ritter, M. (2009). "La complejidad de las organizaciones en el mundo globalizado y el nuevo rol del Dircom”, en J. Costa (eds): Dircom, Estratega de la Complejidad. Nuevos paradigmas para la Dirección de Comunicación. Barcelona, Servei de Publicacions de la Universitat Autónoma de Barcelona, 65-75. Roberts, P. y Dowling, G. (2002). "Corporate Reputation and Sustained Superior Financial Performance", Strategic Management Journal, 23(2), 1077-1093. 
Roos, G., Roos, J., Edvisson, L. \& Dragonetti, N.C. (1997). Intellectual Capital. Navigating in the New Business Landscape. London, McMillan

Schau, H.J. and Gilly, M.C. (2003). "We are what we post? Self-presentation in personal web space", Journal of Consumer Research, 30 (3), 385-404.

Sengupta, P. (1998). "Corporate disclosure quality and the cost of debt", Accounting review, 73(4), 459-474.

Shultz M., Mouritzen J. and Gabrielsen G. (2001). "Sticky Reputation: Analyzing a ranking system", Corporate Reputation Review, 4(1), 24-41.

Singh, J. (2011). "Determinants of effectiveness of Corporate Codes of Ethics: An Empirical Study", Journal of Business Ethics, 101, 385-395. DOI: 10.1007/s10551-010-0727-3.

Sveiby, R.M. (1997). The New Organizational Wealth. San Francisco, Berrett-Koehler

Teece, D.J. (2000). Managing Intellectual Capital. Nueva York, Oxford University Press.

Treviño, L.K., Butterfield, K.D. y McCabe, D.L. (1998). "The ethical context in organizations: Influences on employee attitudes and behavior", Business Ethics Quaterly, 8, 447476.

Verrecchia, R.E. (1983)."Discretional disclosure", Journal of accounting E economics, 5(3), 179-194.

Victor, B. y Cullen, J.B. (1998). "The organizational bases of ethical work climates", Administrative Science Quaterly, 33, 101-125.

Villafañe, J. (2004). La Buena Reputación. Claves del Valor Intangible de las Empresas. Pirámide, Madrid.
Vitell, S. y Ramos, E. (2006). "The impact of Corporate Ethical Values and Enforcement of Ethical codes on the perceived importance of ethics in Business: A comparison of U.S. and Spanish Managers", Journal of Business Ethics, 64, 31-43.

Waddock, S.A. y Graves, S.B. (1997). "The corporate social performance-financial performance link", Strategic Management Journal, 18, 303-319.

Waddock S. (2000). "The multiple bottom lines of corporate citizenship: Social investing, reputation, and responsibility audits", Business and Society Review, 105, 323-345.

Walsh F. y Wiedmann K.P. (2004). "A conceptualization of corporate reputation in Germany: an evaluation and extension of the RQ", Corporate Reputation Review, 6, 304-312.

Wartick S. (2002). "Measuring Corporate Reputation", Business \& Strategy, 41, 371-392.

Watts, R. y Zimmermann, J. (1978). "Towards a positive theory of the determination of accounting standards", Accounting review, 53, 112-134.

Xiao, J. Z., Yang, H, y Chow, C. W. (2004). "The determinants and characteristics of voluntary Internet-based disclosures by listed Chinese companies", Journal of accounting $\mathcal{E}$ public policy, 23, 191-225.

Zabala, I., Panadero, G., Galladord, L.M., Amate, C.M., Sánchez-Galindo, M., Tena, I y Villalba, I. (2005). "Corporate Reputation in Professional Services Firms: Reputation Management Based on Intellectual Capital Management", Corporate Reputation Review, 8(1), 59-71. 